\title{
Improving Sentinel Lymph Node Detection in Patients with Cervical Cancer
}

\author{
Mario M. Leitao Jr., MD ${ }^{1,2}$ \\ ${ }^{1}$ Department of Surgery, Gynecology Service, Memorial Sloan Kettering Cancer Center, New York, NY; ${ }^{2}$ Department of \\ Obstetrics and Gynecology, Weill Cornell Medical College, New York, NY
}

Comprehensive lymphadenectomy for uterine, cervical, and vulvar malignancies has been a mainstay in the upfront surgical treatment of these cancers. The number of lymph nodes retrieved has been a source of pride among gynecologic oncologists, and many have reported that this number has some therapeutic value. However, limited data support a therapeutic benefit to comprehensive lymphadenectomy. In fact, recent randomized trials in uterine cancer reported a lack of survival benefit. ${ }^{1}$

Some key concepts put the potential "therapeutic" role of lymphadenectomy into perspective. One is that we assume that we have removed all possible lymph nodes that are microscopically involved; however, this is unlikely to occur in many cases. There also is an assumption that removal of normal lymph nodes will somehow help the patient survive longer, but data do not support this. In addition to these concepts that challenge the notion of "therapeutic" lymphadenectomy, the potential morbidity that we are inflicting with such lymphadenectomies has been largely ignored. Lymphadenectomy increases operative time, and it puts the patient at risk for lymphocele formation (lymphoceles may become infected and/or symptomatic) as well as lower extremity lymphedema. In fact, lymphadenectomy is associated with an approximately $20 \%$ risk of chronic lower extremity lymphedema. ${ }^{2}$ This can be quite disabling in some women.

While there is a growing sense that lymphadenectomy may not be therapeutic and is definitely associated with morbidity, it is still an important tool for staging and

(C) Society of Surgical Oncology 2015

First Received: 23 June 2015;

Published Online: 9 July 2015

M. M. Leitao Jr., MD

e-mail: leitaom@mskcc.org identifying nodal metastasis. Lymphadenectomy thus provides a more accurate prognosis and helps guide the use of adjuvant therapy. Nodal assessment helps physicians avoid potential overtreatment based on primary tumor features alone, as well as undertreatment in patients with low-risk tumor features but with unsuspected nodal metastasis. This is all still very controversial and many would disagree with these comments.

Sentinel lymph node (SLN) mapping has emerged as the "happy middle ground" in the surgical management of patients with uterine, cervical, and vulvar malignancies. SLN mapping seems to preserve the ability to accurately stage patients while limiting the number of lymph nodes removed, operative time, and morbidity. ${ }^{3}$ Much of the available literature pertains to uterine cancer, but similar findings are reported for SLN mapping in cervical cancer.

The nicely written manuscript from Dr. Imboden and colleagues in this issue is an excellent addition to our growing knowledge regarding SLN mapping in cervical cancer. SLN mapping mostly has been performed using combined technetium-99 and blue dye mapping techniques, as has been done for breast malignancies and melanomas. Successful SLN mapping for cervical cancers must be defined as the ability to identify SLNs in both hemipelvises, because the cervix is a midline organ with known bilateral lymphatic drainage. Successful bilateral SLN detection rates in uterine and cervical cancer using technetium-99 and blue dye are at best $60 \%$. Technetium is not visible; detection is accomplished via an intraoperative gamma probe that uses sound to identify the SLN. Blue dye relies on normal white light to identify SLNs, which can be quite challenging in patients with significant adipose tissue in the retroperitoneum. Therefore, there is an obvious need for improvement in our current SLN mapping techniques. Additionally, technetium-based techniques add cost and patient inconvenience, because they involve injection and 
imaging in nuclear medicine facilities, with the patient awake for some time before the actual surgery.

An alternative to technetium-99 and blue dye is indocyanine green (ICG). ICG is a fluorophobe that fluorescences within the near-infrared spectrum. From personal experience, I can confidently state that SLN mapping is markedly improved using ICG.

Imboden et al. report their detection rate using ICG for SLN mapping in patients with cervical cancer and compare it to their rates using technetium-99 and patent blue dye. As with other studies, their bilateral SLN detection rate with technetium and blue dye was only $61 \%$. This markedly improved to $96 \%$ when they used ICG instead. We also have seen and reported the superiority of ICG in successful bilateral SLN detection and now exclusively use ICGbased SLN mapping for uterine and cervical cancers at our institution. ${ }^{4}$

ICG-based SLN mapping is much more reliable and does not require preoperative injections. It eliminates the cost associated with technetium-based techniques. It most certainly eliminates the patient discomfort and inconvenience. ICG can be found on the formulary of almost all hospitals since it has been in use since the 1940s. Another very interesting finding in the current study was that the false-negative rate was zero. This is quite reassuring when deciding whether SLN mapping is an accurate nodal staging alternative to a more extensive lymphadenectomy.

A limitation to using ICG is the necessity of performing near-infrared (NIR) imaging intraoperatively, which requires additional equipment. NIR imaging is available on the robotic $\mathrm{Si}$ and $\mathrm{Xi}$ surgical platforms. It also is available for nonrobotic laparoscopic and open cases using commercially obtainable equipment. Another limitation is that many of these NIR systems require use of their own ICG instead of the one available on formulary, at a significant added cost. One also must be careful to overly interpret the findings of the current manuscript. It is still a retrospective assessment in a relatively small cohort. However, the findings are consistent with other publications and are very encouraging.

SLN mapping is an emerging approach in the surgical management of patients with uterine and cervical malignancies. It has the potential to completely replace comprehensive lymphadenectomy in the majority of patients. Many questions still need to be addressed before this happens. However, it seems that ICG-based SLN mapping has great promise in optimizing the bilateral SLN detection rates with less cost and patient inconvenience. It is time to reconsider whether nodal counts define surgical quality.

\section{REFERENCES}

1. ASTEC Study Group, Kitchener H, Swart AM, Qian Q, Amos C, Parmar MK. Efficacy of systematic pelvic lymphadenectomy in endometrial cancer (MRC ASTEC trial): a randomized study. Lancet. 2009;373:125-36.

2. Yost KJ, Cheville AL, Al-Hilli MM, et al. Lymphedema after surgery for endometrial cancer: prevalence, risk factors, and quality of life. Obstet Gynecol. 2014;124:307-15.

3. Leitao Jr MM, Khoury-Collado F, Gardner G, et al. Impact of incorporating an algorithm that utilizes sentinel lymph node mapping during minimally invasive procedures on the detection of stage IIIC endometrial cancer. Gynecol Oncol. 2013;129:38-41.

4. Jewell EL, Huang JJ, Abu-Rustum NR, et al. Detection of sentinel lymph nodes in minimally invasive surgery using indocyanine green and near-infrared fluorescence imaging for uterine and cervical malignancies. Gynecol Oncol. 2014;133:274-7. 